\title{
Identification of the translational start site of codon-optimized mCherry in Mycobacterium tuberculosis
}

\author{
Paul Carroll', Julian Muwanguzi-Karugaba' ${ }^{1}$ Eduard Melief ${ }^{2}$, Megan Files $^{2}$ and Tanya Parish ${ }^{1,2^{*}}$
}

\begin{abstract}
Background: Fluorescent proteins are used widely as reporter genes in many organisms. We previously codon-optimized mCherry for Mycobacterium tuberculosis and generated expression constructs with high level expression in mycobacteria with multiple uses in vitro and in vivo. However, little is known about the expression of fluorescent proteins in mycobacteria and the translational start codon for mCherry has not been experimentally determined.

Results: We determined the translational start site for functional (fluorescent) mCherry in mycobacteria. Several potential translational start codons were identified; introduction of downstream stop codons by mutagenesis was used to determine which start codon was utilized in the bacterial cells. Fluorescent protein was expressed from a construct which would allow translation of a protein of 226 amino acids or a protein of 235 amino acids. No fluorescence was seen when a construct which could give rise to a protein of 219 amino acids was used. Similar results were obtained in mycobacteria and in Escherichia coli. Western blotting confirmed that mCherry was expressed from the constructs encoding 235 or 226 amino acids, but not from the plasmid encoding 219 amino acids. N-terminal sequencing and mass determination confirmed that the mature protein was 226 amino acids and commenced with the amino acid sequence AllKE.
\end{abstract}

Conclusion: We conclude that mCherry is expressed in M. tuberculosis as a smaller protein than expected lacking the GFP-derived N-terminal sequence designed to allow efficient fusions.

Keywords: Fluorescence, Gene expression, Mycobacteria, Reporter genes

\section{Background}

Fluorescent proteins (FPs), in particular GFP derivatives, are widely used in mycobacterial systems for many applications [1-13]. Numerous FP variants have been developed, many of which are variants of the original green fluorescent protein (GFP) from the jellyfish Aequorea victoria $[3,14]$, or the red fluorescent protein DsRed from the coral Discosoma sp red $[15,16]$. The wide range of FPs available have different features, including different excitation and emission wavelengths, expression as monomers or dimers, stability, susceptibility to photobleaching, and responsiveness to environmental conditions such as $\mathrm{pH}$ or redox [6,17-22].

\footnotetext{
* Correspondence: tanya.parish@idri.org

${ }^{1}$ Queen Mary University of London, Barts \& The London School of Medicine and Dentistry, Centre for Immunology and Infectious Disease, London, UK ${ }^{2}$ Infectious Disease Research Institute, Seattle, Washington, USA
}

We are interested in the use of FPs as reporters in mycobacteria. We have previously generated FP variants with codon usage optimized for Mycobacterium tuberculosis $[2,13]$. We were able to generate plasmid expression systems with high level constitutive expression that resulted in highly fluorescent strains of use for both in vitro and in vivo studies $[2,13]$. We investigated the use of alternative promoters to generate the highest level of expression possible, without inducing plasmid instability or compromising bacterial growth or virulence $[2,13]$. Such reporter strains are of use in multiple applications, including gene expression studies [2], identifying novel anti-tubercular agents $[12,23,24]$ or monitoring growth in vivo [13].

The level of expression of an FP is dependent on transcriptional and translational factors, but in bacteria the main factor is the promoter. The promoter location in 
relation to the transcriptional and translational start site can play a role in determining overall expression levels. In our original constructs we used the translational start site of the engineered protein mCherry from the literature in order to place the gene in the correct location relative to the promoter. The original development of mCherry involved replacing the first seven amino acids to improve the ability to generate functional $\mathrm{N}$-terminal fusions, although the sequence still retained a downstream methionine which could function as an alternative start [17]. In order to generate further improved constructs and to characterize the recombinant fluorescent protein expressed in mycobacteria, we determined the functional translational start site for mCherry. Our data demonstrate that mCherry is expressed in $M$. tuberculosis as a truncated protein which lacks the $\mathrm{N}$-terminal sequence derived from GFP (MVSKGEE).

\section{Results and discussion}

We are interested in the use of FPs as reporters of bacterial viability and gene expression in mycobacteria, in particular in M. tuberculosis [2,13]. The expression of FPs at high level can be detrimental to bacterial viability due to the metabolic burden it places on the cell. We have developed codon-optimized FPs for M. tuberculosis to, at least partly, overcome this by removing rare codons and allowing for high level expression [2].

\section{The functional translational start site for mCherry in M. tuberculosis}

We previously optimized expression of mCherry by codon-optimization of the most commonly used variant [2]. During our studies we noted that there were several potential translational start sites. Three of these (including the expected one) had a ribosome binding site (RBS) motif directly upstream, suggesting that translation could initiate in multiples places (Figure 1). Interestingly, the two internal RBS and the downstream start codons were present in the original sequence and were not introduced by codon optimization. The translational start site of DsRed, from which mCherry is derived, has not been determined functionally for the native protein [15]. In the original studies the DsRed protein was expressed with a His-Tag and the primary amino acid sequence was derived from the sequence of the mRNA rather than protein sequencing [15]. mCherry itself was constructed by replacing the first seven amino acids of mRFP1.1 with the corresponding GFP sequence and is thus a fusion protein. Therefore it is possible that the functional protein is shorter than that expected, or that different proteins could be produced in different species depending on the efficiency of the RBS and the translational machinery.

We decided to determine which of the alternative translational start sites are functional in $M$. tuberculosis.
We used an expression construct (pCherry10) in which mCherry is under the control of the G13 promoter from M. marinum [13]; this is a strong constitutive promoter. There are three potential translational start sites at $+1,+28$ and +52 (relative to the expected start site); to determine which of these was functioning in mycobacteria, we introduced a stop codon either at +13 or at +40 (plasmids pCherry 29 and pCherry30). This would prevent translation of full length protein from the +1 or +28 start sites respectively and translation would results in proteins of 226 or 219 amino acids, instead of 235 in the "full length" version.

Constructs were introduced into M. tuberculosis. We already demonstrated that functional fluorescent protein was produced from the parental vector pCherry10 [13]. It was apparent immediately that functional FP was made from one of the expression vectors, since the colonies were visibly colored (Figure $2 \mathrm{~A}$ ); this was confirmed by measuring fluorescence in cells (Figure 2B). No difference in fluorescence intensity was seen when a stop codon was introduced upstream of the start codon at +28 , whereas a complete loss of fluorescence was noted when a stop codon was introduced upstream of the +52 start codon. These data confirm that functional, fluorescent protein is obtained in M. tuberculosis from a truncated protein $\left(\mathrm{mCherry}_{226}\right)$, but not from mCherry ${ }_{219}$.

The same results were obtained in Escherichia coli (data not shown), where fluorescence was seen with mCherry $_{226}$, but not mCherry $_{219}$. This suggested that the functional translational start site was the same in both species. In order to determine if the lack of fluorescence was from lack of expression or if the protein was produced, but not functional, we looked at protein levels by Western blotting. Expression of mCherry was seen from plasmid pCherry 10 and pCherry29, but not from pCherry30, suggesting that the translational start site at +52 does not lead to the production of protein (Figure 2C).

\section{$\mathrm{mCherry}$ is expressed as a mature protein of $\mathbf{2 2 6}$ amino acids}

The mutagenesis study suggested that mCherry could be expressed as a functional 226 amino acid protein from pCherry29, but did not exclude the possibility that it was expressed as a longer protein form the pCherry10 plasmid. In order to address this, we purified mCherry from an $M$. tuberculosis transformant carrying pCherry10. Mass determination suggested that the protein had a size of 25612.2 Da, which closely approximated the predicted size for the 226 amino acid protein of 25579.9 Da (Figure 3). $\mathrm{N}$-terminal sequencing confirmed that the purified protein commenced with the amino acids AAIKE, confirming that mCherry226 is the functional species of protein found in mycobacteria. 


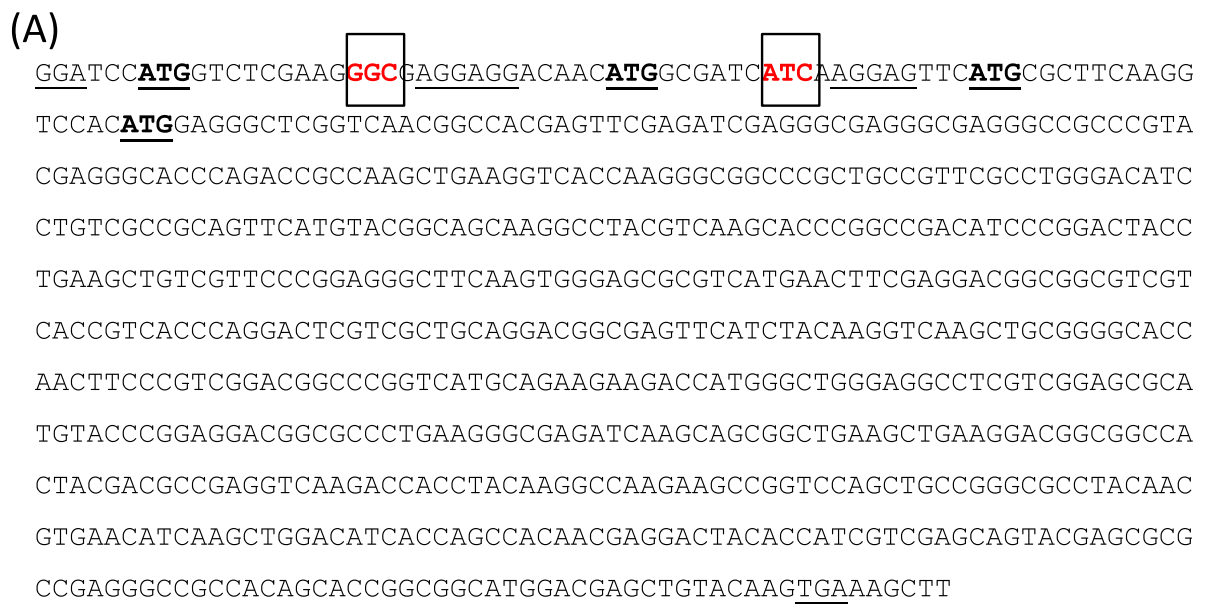

(B)

MVSKGEEDNMAI IKEFMRFKVHMEGSVNGHEFEIEGEGEGRPYEGTQTAKLKVTKGGP
LPFAWDILSPQFMYGSKAYVKHPADI PDYLKLSFPEGFKWERVMNFEDGGVVTVTQDS
SLQDGEFIYKVKLRGTNFPSDGPVMQKKTMGWEASSERMYPEDGALKGEIKQRLKLKD
GGHYDAEVKTTYKAKKPVQLPGAYNVNIKLDITSHNEDYTIVEQYERAEGRHSTGGMD
ELYK

Figure 1 Sequence of mCherry. (A) DNA sequence of codon-optimized mCherry gene. The potential translational start sites are indicated in bold and underlined; potential ribosome-binding sites and the stop codon are underlined. The codons mutagenized to stop codons are in red and boxed. (B) Protein sequence of mCherry indicating the four potential start sites in bold. The identified start site is underlined.

\section{Conclusions}

We obtained high-level expression of mCherry from the G13 promoter in M. tuberculosis. Analysis of the mature protein expressed in $M$. tuberculosis confirmed that the translational start site at +28 was utilized and the $\mathrm{N}$ terminal sequence of the mature protein was AIIKE. The expression of truncated mCherry gave rise to highly fluorescent colonies, confirming that this truncated protein is functional. However, the absence of the GFP-derived peptide designed to allow efficient $\mathrm{N}$-terminal fusions could have a negative impact on stability and function of fusion proteins.

\section{Methods}

\section{Bacterial culture}

Escherichia coli DH5 $\alpha$ was cultured in LB medium or on LA agar. M. tuberculosis $\mathrm{H} 37 \mathrm{Rv}$ was grown in Middlebrook 7H9 medium plus $10 \% \mathrm{v} / \mathrm{v}$ OADC (oleic acid, albumen, dextrose, catalase) supplement (Becton Dickinson) and $0.05 \% \mathrm{w} / \mathrm{v}$ Tween 80 or on Middlebrook $7 \mathrm{H} 10$ agar

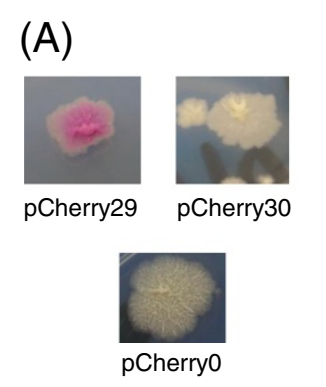

(B)

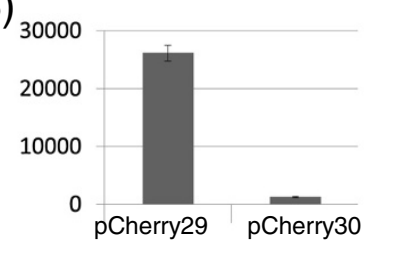

(C)

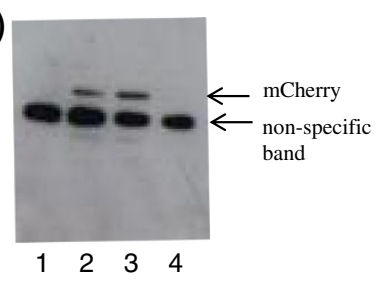

Figure 2 Identification of the translational start site of $\mathbf{m C h e r r y ~ i n ~} \mathbf{M}$. tuberculosis. Plasmids carrying mCherry were electroporated into $M$. tuberculosis and transformants selected on solid medium using hygromycin. The predicted proteins expressed from each plasmid are - pCherry 29 $=\mathrm{mCherry}_{226 ;}$ pCherry30 = mCherry $219 ; \mathrm{pCherry0}$ (control plasmid) = no mCherry expression. (A) Transformant colonies. (B) Fluorescence was measured in liquid culture. Cultures were measured at Ex587/Em610 and results are expressed as relative fluorescence units (fluorescence/OD). Data are the mean and standard deviation from three independent transformants $\mathbf{( C )}$ Western analysis of protein expression in E. coli. Cell-free extracts were generated from transformants carrying plasmids and probed with anti-mCherry antibodies. Lane 1: no plasmid. Lane 2- pCherry 10 (mCherry 235 ). Lane 3- pCherry29 (mCherry ${ }_{226}$ ). Lane 4- pCherry30 (mCherry $\left.{ }_{219}\right)$. A non-specific band reacting with the commercial antibody was seen in all lanes, including E. coli lacking a plasmid. 

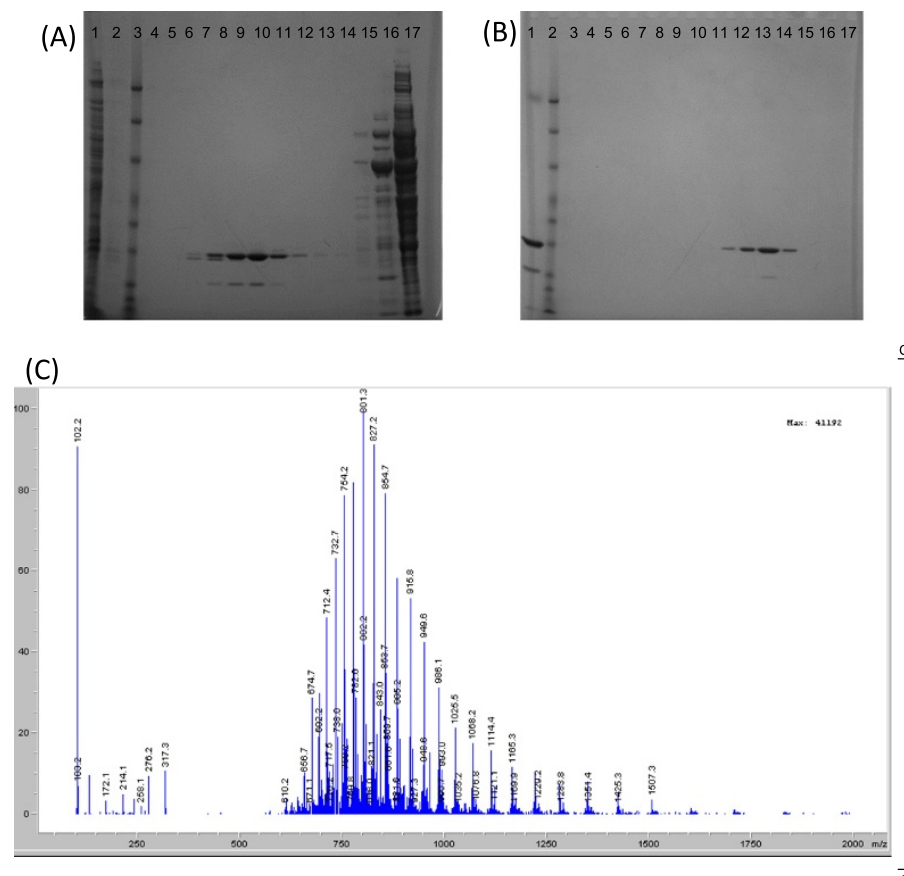

AIIKEFMRFKVHMEGSVNGHEFEIEGEGEGRPYEGTQTAKLKVTKGGPLPFAWDILSPQFMYGSKAYVKHPADIPDYLKLSF PEGFKWERVMNEEDGGVVTVTQDSSLQDGEFIYKVKLRGTNFPSDGPVMQKKTMGWEASSERMY PEDGALKGEIKQRLKLKD
GGHYDAEVKTTYKAKKPVQLPGAYNVNIKLDITSHNEDYTIVEQYERAEGRHSTGGMDELYK
25579.9 Da

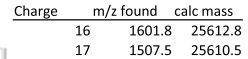

$\begin{array}{lll}16 & 1601.8 & 25612.8 \\ 17 & 1507.5 & 25610.5 \\ 18 & 142.9 & 2561.2\end{array}$ 1423.925612 .2 $1349.2 \quad 25615.8$ 1281.525610 .0 $\begin{array}{lll}1220.5 & 25609.5 \\ -165.3 & 25614.6\end{array}$ 1114.525610 .5 $025.4 \quad 25610$ $986.1 \quad 25612.6$ $949.6 \quad 25612$ 915.625608 .8 $884.2 \quad 25612.8$ $854.7 \quad 25611.0$ $827.2 \quad 25612.2$ 877.125612 .8 $754.3 \quad 25612.2$ $732.9 \quad 25616.5$ $712.5 \quad 25614$ 693.325615. 674.925608 .2 657.925619 $641.3 \quad 25612$. $\begin{array}{lrr} & 41 & 625.6 \quad 25608.6 \\ \text { Average } & 25612.2 & \pm 2.6\end{array}$

Figure 3 Purification and mass determination of mCherry. (A) 4-12\% Bis-Tris SDS-PAGE gel of Q-sepharose purification. Lane 1 - cleared cell-free extract; Lane 2- wash; Lane 3 - Benchmark ladder; Lane 4 to 17 - gradient elutions. (B) 4-12\% Bis-Tris SDS-PAGE of size exclusion purification. Lane 1- loaded protein; Lane 2 - Benchmark ladder; Lanes 3 to 17 - elution fractions. (C) ESI-MS ion envelope of purified mCherry protein, deconvolution calculation, and primary sequence of mCherry protein based on observed mass and protein sequencing. Amino-acids detected from $\mathrm{N}$-terminal protein sequencing in bold, chromophore forming residues underlined, with the expected mass of the mature protein listed after the primary sequence.

(Becton Dickinson) plus 10\% v/v OADC. Hygromycin was used at $100 \mu \mathrm{g} / \mathrm{ml}$ where required.

\section{Construction of expression vectors}

The mCherry expression vector pCherry 10 was used as a template for mutagenesis [13]. Site directed mutagenesis was used to introduce a stop codon using primer pair CherrySTOPA1 5'- TCC ATG GTC TCG AAG TGA GAG GAG GAC AAC ATG-3' and CherrySTOPA2 5'CAT GTT GTC CTC CTC TCA CTT CGA GAC CAT GGA -3 ' to generate pCherry29, or primer pair CherrySTOPB1 5'-GAC AAC ATG GCG ATC TGA AAG GAG TTC ATG CGC-3' and CherrySTOPB2 5'- GCG CAT GAA CTC CTT TCA GAT CGC CAT GTT GTC-3' to generate pCherry30. Stop codons are in bold.

\section{Quantitation of fluorescence in whole cells}

M. tuberculosis was electroporated as described previously [25] and transformants selected with hygromycin. E. coli and $M$. tuberculosis were grown to stationary phase, harvested, washed twice in $10 \mathrm{mM}$ Tris $\mathrm{pH} 8.0$ and resuspended in $10 \mathrm{mM}$ Tris $\mathrm{pH} 8.0$ to an $\mathrm{OD}_{580}$ of 0.25 , $0.10,0.05$ and 0.01 in $12 \times 100 \mathrm{~mm}$ glass culture tubes. Fluorescence was measured on a Shimadzu RF-1501 spectrofluorimeter (Shimadzu) with a detection range of 0-1015 relative fluorescent units at the emission and excitation wavelengths of 587/610 nm.

\section{Western analysis of fluorescent proteins}

Cell extracts were prepared from liquid cultures. Cells were harvested by centrifugation, washed twice in $10 \mathrm{mM}$ Tris ( $\mathrm{pH}$ 8.0), resuspended in $1 \mathrm{~mL}$ of $10 \mathrm{mM}$ Tris ( $\mathrm{pH}$ 8.0), and added to lysing matrix B tubes (QBiogene). Cells were disrupted using the Fastprep (QBiogene) set at speed 6.0 for 30 seconds. Samples were centrifuged for two min, and the supernatant was recovered and filter sterilized. Protein was quantified using a BCA kit (Pierce), and $10 \mu \mathrm{g}$ of total protein was subjected to Western blot using rabbit anti-mCherry antibody (Clontech). The primary antibody was detected using horseradish peroxidase goat-anti-rabbit (Sigma), and activity was detected using an ECL kit (GE Healthcare).

\section{Mass determination and $\mathrm{N}$-terminal sequencing of mCherry} Cell-free extracts were prepared from recombinant $M$. tuberculosis carrying plasmid pCherry10, diluted 5 fold into $20 \mathrm{mM}$ Tris $\mathrm{pH} 8.0,10 \mathrm{mM} \mathrm{NaCl}$ and loaded onto a buffer equilibrated Q-sepharose column (GE Healthcare). 
The column was washed with 10 column volumes of $20 \mathrm{mM}$ Tris $\mathrm{pH}$ 8.0, $10 \mathrm{mM} \mathrm{NaCl}$ and mCherry protein was eluted using a stepwise increase in salt concentration up to $1 \mathrm{M} \mathrm{NaCl}$. Eluted fractions were concentrated separately using Amicon Ultra Centrifugal Filters 10,000 MWCO (Millipore), coloured fractions pooled, and concentrated to $100 \mu \mathrm{L}$. Protein was further purified by applying pooled fractions to a Superdex 75 size-exclusion column (GE Healthcare) and eluting with $20 \mathrm{mM}$ Tris $\mathrm{pH}$ 8.0, $10 \mathrm{mM}$ $\mathrm{NaCl}$ buffer. Coloured fractions were pooled and concentrated to $25 \mu \mathrm{M}(0.62 \mathrm{mg} / \mathrm{mL})$. Protein was analyzed by LC-ESI-MS by diluting $15 \mu \mathrm{L}$ of concentrate into $15 \mu \mathrm{L}$ acetonitrile, $0.1 \%$ TFA, loading onto a Polaris 3 C8-A $150 \times 4.6 \mathrm{~mm}$ column and eluting with an acetonitrile gradient over $30 \mathrm{~min}$. Mass peaks from multiply charged species were deconvoluted to yield the mass of the mCherry protein. Edman degradation was carried out on 400 pmol of purified protein by Biosynthesis, Inc. on a Procise II Protein Sequencing System (Applied Biosystems).

\section{Competing interests}

The authors declare that they have no competing interests.

\section{Authors' contributions}

$\mathrm{PC}, \mathrm{JM}, \mathrm{EM}$ and $\mathrm{MF}$ conducted the experimental work. PC, JM, EM and TP analyzed the data. TP, EM and PC wrote the paper. JM and MF provided input for the paper. All authors read and approved the final manuscript.

\section{Acknowledgements}

We thank Amanda Brown and Lise Schreuder for technical assistance and helpful discussion. This work was supported by the Bill and Melinda Gates Foundation grant OPP42786.

Received: 21 October 2013 Accepted: 6 June 2014

Published: 17 June 2014

\section{References}

1. Carroll P, James J: Assaying promoter activity using LacZ and GFP as reporters. In Mycobacteria Protocols. Edited by Parish T, Brown AC. New York: Humana Press; 2009:265-278. Methods in Molecular Biology.

2. Carroll P, Schreuder LJ, Muwanguzi-Karugaba J, Wiles S, Robertson BD, Ripoll J, Ward TH, Bancroft GJ, Schaible UE, Parish T: Sensitive detection of gene expression in mycobacteria under replicating and non-replicating conditions using optimized far-red reporters. PLoS One 2010, 5:e9823.

3. Chalfie M, Tu Y, Euskirchen G, Ward WW, Prasher DC: Green fluorescent protein as a marker for gene expression. Science 1994, 263:802-805.

4. Cosma CL, Humbert O, Ramakrishnan L: Superinfecting mycobacteria home to established tuberculous granulomas. Nat Immunol 2004, 5:828-835.

5. Cosma CL, Humbert O, Sherman DR, Ramakrishnan L: Trafficking of superinfecting Mycobacterium organisms into established granulomas occurs in mammals and is independent of the Erp and ESX-1 mycobacterial virulence loci. J Infect Dis 2008, 198:1851-1855.

6. Darby CM, Ingolfsson HI, Jiang X, Shen C, Sun M, Zhao N, Burns K, Liu G, Ehrt S, Warren JD, Anderson OS, Brickner SJ, Nathan C: Whole cell screen for inhibitors of $\mathrm{pH}$ homeostasis in Mycobacterium tuberculosis. PLOS One 2013, 8:e68942.

7. Dhandayuthapani S, Via LE, Thomas CA, Horowitz PM, Deretic D, Deretic V: Green fluorescent protein as a marker for gene expression and cell biology of mycobacterial interactions with macrophages. Mol Microbiol 1995, 17:901-912.

8. Gall K, Barker LP: Differential green fluorescent protein expression from mycobacterial promoter constructs in Escherichia coli and Mycobacterium marinum. FEMS Microbiol Lett 2006, 255:301-307.
9. Golchin SA, Stratford J, Curry RJ, McFadden J: A microfluidic system for long-term time-lapse microscopy studies of mycobacteria. Tuberculosis 2012, 92:489-496.

10. Kaps I, Ehrt S, Seeber S, Schnappinger D, Martin C, Riley LW, Niederweis M: Energy transfer between fluorescent proteins using a co-expression system in Mycobacterium smegmatis. Gene 2001, 278:115-124.

11. Kremer L, Baulard A, Estaquier J, Poulain-Godefroy O, Locht C: Green fluorescent protein as a new expression marker in mycobacteria. Mol Microbiol 1995, 17:913-922.

12. Ollinger J, Bailey M, Casey A, Florio S, Alling T, Moraski GC, Miller MJ, Parish T: A dual read-out assay to evaluate the potency of compounds active against Mycobacterium tuberculosis. PLoS One 2013, 8:e60531.

13. Zelmer A, Carroll P, Andreu N, Hagens K, Mahlo J, Redinger N, Robertson BD, Wiles S, Ward TH, Parish T, Ripoll J, Bancroft GJ, Schaible UE: A new in vivo model to test anti-tuberculosis drugs using fluorescent imaging. J Antimicrob Chemother 2012, 67:1948-1960.

14. Tsien RY: The green fluorescent protein. Annu Rev Biochem 1998, 67:509-544.

15. Matz MV, Fradkov AF, Labas YA, Savitsky AP, Zaraisky AG, Markelov ML, Lukyanov SA: Fluorescent proteins from nonbioluminescent Anthozoa species. Nat Biotechnol 1999, 17:969-973.

16. Gross LA, Baird GS, Hoffman RC, Baldridge KK, Tsien RY: The structure of the chromophore within DsRed, a red fluorescent protein from coral. Proc Natl Acad Sci USA 2000, 97:11990-11995.

17. Shaner NC, Campbell RE, Steinbach PA, Giepmans BN, Palmer AE, Tsien RY: Improved monomeric red, orange and yellow fluorescent proteins derived from Discosoma sp. red fluorescent protein. Nat Biotechnol 2004 22:1567-1572.

18. Muller-Taubenberger $\mathrm{A}$, Anderson Kl: Recent advances using green and red fluorescent protein variants. Appl Microbiol Biotechnol 2007, 77:1-12.

19. Miyawaki A, Shcherbakova DM, Verkhusha W: Red fluorescent proteins: chromophore formation and cellular applications. Curr Opin Struct Biol 2012, 22:679-688.

20. Morris MC: Fluorescent biosensors - probing protein kinase function in cancer and drug discovery. Biochim Biophysic Acta 1834, 2013:1387-1395.

21. Lukyanov KA, Belousov W: Genetically encoded fluorescent redox sensors. Biochim Biophysic Acta 2013, 1840:745-756.

22. Poea-Guyon S, Pasquier H, Merola F, Morel N, Erard M: The enhanced cyan fluorescent protein: a sensitive $\mathrm{pH}$ sensor for fluorescence lifetime imaging. Anal Bioanal Chem 2013, 405:3983-3987.

23. Kumar D, Raj KK, Bailey M, Alling T, Parish T, Rawat DS: Antimycobacterial activity evaluation, time-kill kinetic and 3D-QSAR study of C-(3-aminomethyl-cyclohexyl)-methylamine derivatives. Bioorg Med Chem Lett 2013, 23:1365-1369.

24. Moraski G, Markley LD, Cramer J, Hipskind PA, Boshoff H, Bailey M, Alling T, Ollinger J, Parish T, Miller MJ: Advancement of imidazo[1,2-a]pyridine-3carboxamides with improved pharmacokinetics nanomolar activity against Mycobacterium tuberculosis. ACS Med Chem Lett 2013, 4:675-679.

25. Parish T, Stoker NG: Electroporation of mycobacteria. In Mycobacteria Protocols, Volume 101. Edited by Parish T, Stoker NG. Totowa: Humana Press; 1998:129-144.

doi:10.1186/1756-0500-7-366

Cite this article as: Carroll et al:: Identification of the translational start site of codon-optimized mCherry in Mycobacterium tuberculosis. BMC Research Notes 2014 7:366. 